\title{
Experimental Demonstration of User Allocation in a Subcarrier Multiplexing-Based Multiuser LiFi System
}

\author{
Mounir Mohammedi Merah \\ Laboratoire d'Ingénierie des Systèmes de \\ Versailles \\ UVSQ, Université Paris-Saclay \\ Vélizy, France \\ mounir.mohammedi-merah@lisv.uvsq.fr
}

\author{
Luc Chassagne \\ Laboratoire d'Ingénierie des Systèmes de \\ Versailles \\ UVSQ, Université Paris-Saclay \\ Vélizy, France \\ luc.chassagne@uvsq.fr
}

\author{
Hongyu Guan \\ Laboratoire d'Ingénierie des Systèmes de \\ Versailles \\ UVSQ, Université Paris-Saclay \\ Vélizy, France \\ hongyu.guan@lisv.uvsq.fr
}

\begin{abstract}
We want to allocate the users to the optimal subcarriers in visible light communication. Allocation algorithms adapted from fiber-optic and implemented in an experimental setup attain a deviation from the bit rate target inferior to 5 percent for 11 users.
\end{abstract}

Keywords-Indoor network, multiuser, visible light communication, multiband CAP, subcarrier multiplexing, LiFi

\section{INTRODUCTION}

While working towards $5 \mathrm{G}$ and beyond with light-fidelity (LiFi) the work presented here focuses on the implementation of a multi-access visible light communication (VLC) system. We associated multiband carrierless amplitude and phase (multiband CAP, m-CAP) modulation and subcarrier multiplexing $(\mathrm{SCM})$ in a realistic experimental setup in order to provide a flexible multi-user implementation. Multiband CAP is a high spectral efficiency modulation with high flexibility with the ability to use bit and power loading [1]. The scheme is associated with SCM [2] to provide multi-user access with little computational cost.

Our previous work [3] was geared towards the optimization of the throughput performances by using different transmit filters parameters for each subcarrier. Following this work, employing a white light-emitting device (LED) and a silicon photodiode, we achieved a flexible 1 to 20 users VLC system with a $5.08 \mathrm{~b} / \mathrm{s} / \mathrm{Hz}$ spectral efficiency for a total data rate of
$160 \mathrm{Mb} / \mathrm{s}$, over a 2.15 meter distance.

The problematic that we focus on here is how to allocate the users to the subcarriers. In order to associate each users to the subbands optimally, multiple allocation algorithms are explored by evaluating their performances with the experiment setup. The conditions at which the algorithms operate change in VLC compared with fiber-optic communications, where SCM is mainly used [2]. For VLC, a lower amount of total subcarriers can be allocated and higher variations in signal-tonoise ratio (SNR) are present [4][5]. As such, allocation algorithms for SCM need to be adapted and compared to an original scheme found in fiber communications [6].

\section{EXPERIMENT SETUP}

In the work presented here, a commercial white LED downlight, typically found in museum or hospitals, is used as the transmitter for the VLC system. The modulation process of $\mathrm{m}$-CAP is associated with a pre-equalization technique to extend the available modulation bandwidth. At the receiver, which can represent any user, a $25 \mathrm{~mm}$ biconvex lens, a photodetector, and a transimpedance amplifier circuit are used to detect the signal. The distance between the transmitter and receiver is set at 2.15 meter with line of sight (LOS) for a realistic setup. The information of which subcarrier or group of subcarriers a user must demodulate to recover its data is stored in the first subcarrier. Figure 1 shows a schematic of the
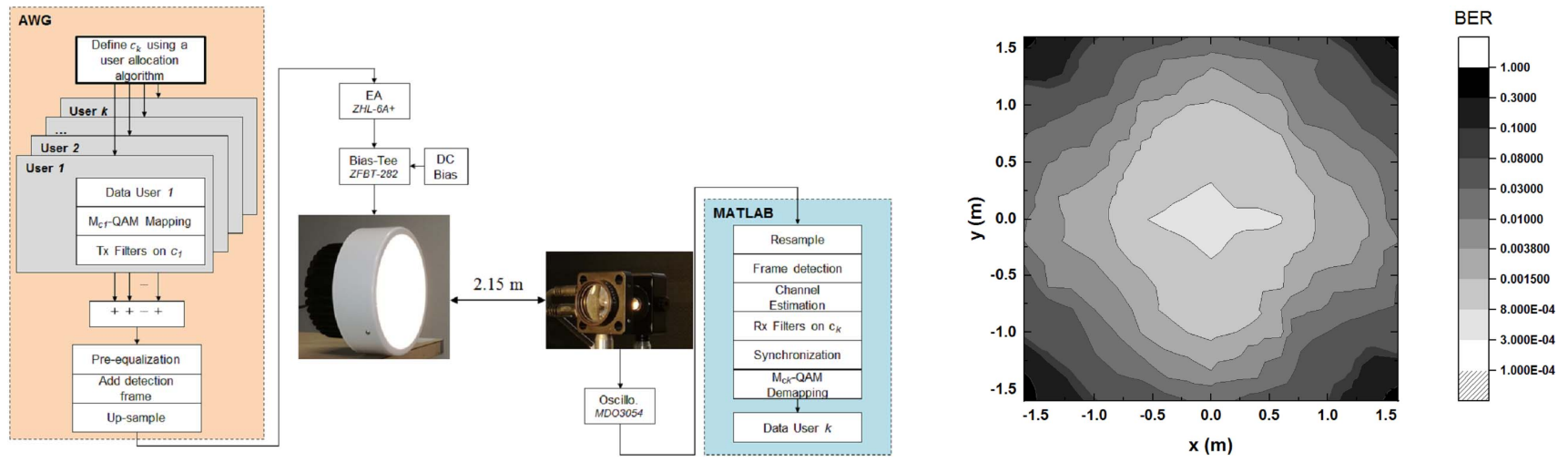

Fig. 1: Schematic and photo of the experiment setup (left). The LED is a commercially available white LED downlight with a $1.4 \mathrm{MHz}$ modulation bandwidth, extended with pre-equalization. 3D measured bit error rate (right) with the receiver at a 2.15 meter plane from the transmitter. 
experimental setup and the 3D bit error rate (BER).

\section{ALGORITHMS}

We define $\mathrm{k}$ as the number of bits that each constellation symbol corresponds to. Each user has a data rate target, defined as $\mathrm{d}_{\mathrm{OBJ}}$ and the aim of the algorithms is to get as close as possible to that objective, knowing that the number of subcarriers to be allocated is limited. The associated $\mathrm{k}$ is called $\mathrm{k}_{\mathrm{OBJ}}$. Four algorithms are investigated. Performances are estimated with the attained deviation from the data rate target. The sum of the target throughputs for each user is equal to the total data rate provided by the system.

The first algorithm is a direct static allocation where the average center frequency of all the allocated subcarriers to a specific user is close to the center of the total signal bandwidth. The aim is to ensure equal quality of service (QoS) for each user in terms of SNR. This scheme also have low implementation cost and doesn't need to be recalculated every time the bit loading system changes the modulation order of a subcarrier. The second algorithm [6] is a SCM allocation scheme first found in OFDM and fiber-optic communication. It is recalculated when the bit loading system changes the modulation order of a subcarrier. The third algorithm, named 'proposed method 1', is based on the second one and is adapted to the conditions of large swings in SNR in a VLC channel. The forth algorithm, named 'proposed method 2', is the same as the previous one except that QoS for each user is considered in the same way as the first algorithm.

\section{RESULTS}

Performances of each algorithm are estimated against the total number of users that needs to be allocated (Figure 3) and against the total number of subcarriers in the multiband CAP modulation scheme (Figure 4). The deviation from the data rate measured is an average of the deviation measured for each user. The target defined for each user is a random throughput with the only condition being that the sum of all targets doesn't exceed the total throughput provided by the transmitter. The deviation is equal to $0 \%$ if the target data rate is attained or exceeded. When the throughput for the user is below the objective, we define the deviation as the absolute difference between the attained data rate and the data rate targeted in percent.

In figure 3, the proposed algorithm 1 has the best performances being able to provide up to 11 users with a deviation smaller than $5 \%$. At the same condition, the fiberoptics algorithm is only able to provide up to 4 users. In figure 4 , the proposed algorithm 1 obtains the best performances but the higher the number of subcarriers, the less difference between each scheme there is. In consequence, the proposed methods only outperform the fiber-optic algorithm with a low amount of subcarrier, which corresponds to our m-CAP VLC scenario.

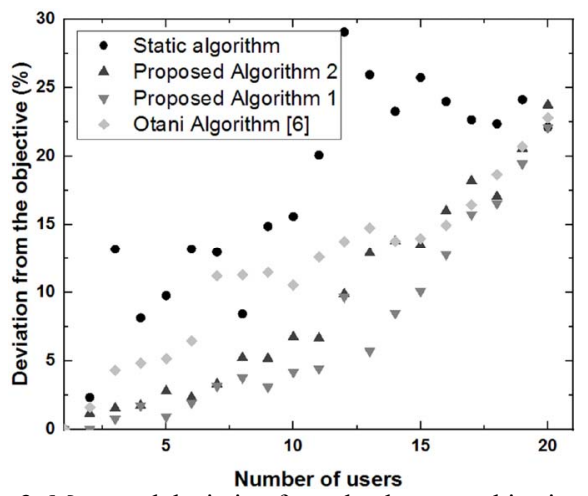

Fig. 3: Measured deviation from the data rate objective for each user versus the number of users using all four algorithms. A 20-CAP signal is used and 1 to 20 users are assigned using the algorithms.

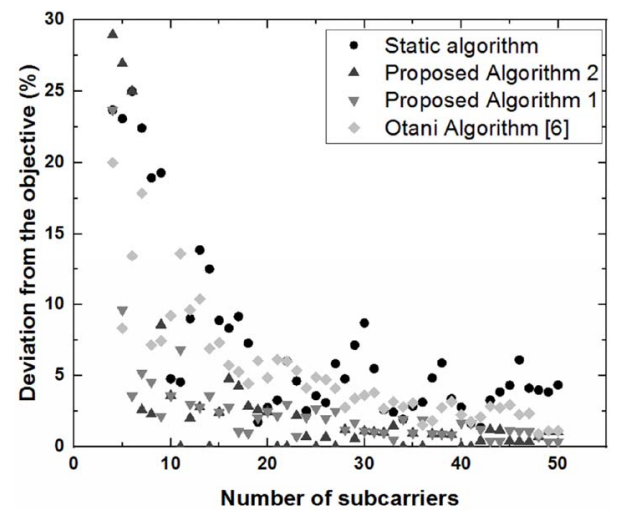

Fig. 4: Measured deviation from the data rate objective for each user versus the number of subcarriers using all four algorithms in a 4 users scenario.

\section{ACKNOWLEDGMENT (Heading 5)}

The authors thank the OLEDCOMM-LISV industrial chair that provides funding for the experiments.

\section{REFERENCES}

[1] M. I. Olmedo et al., "Multiband Carrierless Amplitude Phase Modulation for High Capacity Optical Data Links," in Journal of Lightwave Technology, vol. 32, no. 4, pp. 798-804, Feb.15, 2014.

[2] Rongqing Hui, Benyuan Zhu, Renxiang Huang, C. T. Allen, K. R. Demarest and D. Richards, "Subcarrier multiplexing for high-speed optical transmission," in Journal of Lightwave Technology, vol. 20, no. 3, pp. 417-427, Mar 2002.

[3] M. M. Merah, H. Guan and L. Chassagne, "Performance optimization in multi-user multiband carrierless amplitude and phase modulation for visible light communication," 2018 Global LIFI Congress (GLC), Paris, France, 2018, pp. 1-4.

[4] Chao Li, Qi Yang, "Optical OFDM/OQAM for the Future Fiber-optics Communications," Procedia Engineering, Vol. 140, 2016, pp. 99-106.

[5] M. S. Ab-Rahman, N. I. Shuhaimi, L. A. Azizan and M. R. Hassan, "Analytical Study of Signal-to-Noise Ratio for Visible Light Communication by Using Single Source," in Journal of Computer Science, vol. 8, no. 1, pp. 141-144, 2012.

[6] Y. Otani, S. Ohno, K. a. Donny Teo and T. Hinamoto, "Subcarrier Allocation for multi-user OFDM system," 2005 Asia-Pacific Conference on Communications, Perth, WA, 2005, pp. 1073-1077. 